\title{
Inulinase ( $\beta$-fructofuranosidase) production by Kluyveromyces marxianus in batch culture
}

\section{Sarad Parekh* and Argyrios Margaritis}

Chemical and Biochemical Engineering

Faculty of Engineering Science, The University of Western Ontario, London, Ontario, Canada N6A 5B9

Appl Microbiol Biotechnol (1985) 22:446-448

In the title and throughout the paper the correct spelling of the genus should have read Kluyveromyces 